\title{
TABLA DE FACTORES DE LA LEY DE ISAPRES Y DERECHOS FUNDAMENTALES (TRIBUNAL CONSTITUCIONAL)
}

\author{
Comentario de Pablo Marshall Barberán
}

Sentencia Tribunal Constitucional (extracto), rol 1710-10

Santiago, 6 de agosto de 2010

$[\ldots]$

\section{LOS DERECHOS CONSTITUCIONALES AFECTADOS POR EL PRECEPTO LEGAL BAJO EXAMEN DE INCONSTITUCIONALIDAD.}

OCTOGESIMOCUARTO: Que los derechos que se encuentran consagrados en las disposiciones constitucionales consideradas trasgredidas por las sentencias previas de inaplicabilidad que dan sustento a este proceso, sólo pueden ser descritos si se enmarcan en su sustrato normativo constitucional -en la dignidad de la persona- y en su pertenencia a la calidad de derechos fundamentales;

$[\ldots]$

NONAGESIMOQUINTO: Que los derechos fundamentales sociales no sólo se han consolidado en su establecimiento normativo y en su garantización positiva, sino que en el actual debate jurisdiccional constitucional se discute su justiciabilidad, precisamente a partir de casos de revisiones judiciales del derecho a la salud acaecidos en varios países (Rodolfo Figueroa García Huidobro: Justiciabilidad de los derechos económicos, sociales y culturales. Discusión teórica, en: Revista Chilena de Derecho, 36, 3, 2009, pp. 587-620);

\section{EL DERECHO DE IGUALDAD ANTE LA LEY.}

[...]

NONAGESIMOSÉPTIMO: Que la consagración de la igualdad ante la ley en la Constitución chilena, en los términos vigentes transcritos, responde a una tradición de nuestros textos constitucionales, incluyendo algunas referencias del número $1^{\circ}$ del artículo 10 de la Carta de 1925. Sin embargo, el precepto constitucional hoy vigente contiene dos disposiciones que han ampliado y profundizado el contenido de esta garantía y que se vinculan directamente con al asunto que esta Magistratura resuelve en autos: a) La consagración de la igualdad ante la ley de hombres y mujeres, y b) La prohibición a la ley y a la autoridad de establecer diferencias arbitrarias; 


\section{$[\ldots]$}

CENTÉSIMO: Que en el fallo Rol 1273, recaído en causa de inaplicabilidad por inconstitucionalidad que sirve de sustento al procedimiento de autos, esta Magistratura incorporó en su entendimiento sobre la igualdad los criterios jurisprudenciales que en el derecho comparado se han utilizado en la última década para afrontar las crecientes complejidades que presentan los casos sobre tal garantía constitucional, así como los afanes desplegados por la doctrina en la misma dirección. De estos aportes, entre otros descritos en la sentencia citada, destaca el enfoque alemán que distingue conceptualmente entre "igualdades esenciales" y "desigualdades esenciales", de tal modo que estamos en presencia de una igualdad esencial cuando "personas, grupos de personas o situaciones, sobre la base de un punto de partida (tertium comparationis), son comparables", de lo que, consecuentemente, el Tribunal Constitucional Federal alemán ha decidido que la Ley Fundamental considera arbitrario y, por ende, inconstitucional, tratar desigualmente a las igualdades esenciales, así como tratar igualmente a las desigualdades esenciales. Además, se agrega la denominada "nueva fórmula", consistente en considerar lesionada la igualdad ante la ley cuando un grupo de destinatarios de la norma, comparados con otro grupo de destinatarios de la norma, son tratados de manera distinta, a pesar de que entre ambos grupos no media ninguna diferencia de tal entidad o importancia que pudiera justificar un tratamiento desigual. Para poder dimensionar tales situaciones, esta fórmula requiere expresamente una ponderación en el sentido de examen de proporcionalidad, especialmente respecto de una diferencia de trato de gran intensidad, para lo cual se requiere que aquélla persiga un fin legítimo, que esa búsqueda sea necesaria y que presente una razonable relación con el valor del fin propuesto";

\section{LA IGUALDAD ENTRE EL HOMBRE Y LA MUJER.}

[...]

De todo lo expuesto es nítido que la igualdad de derechos del hombre y de la mujer es una categoría estándar en las legislaciones nacionales e internacionales, según la medida de equiparación de los derechos de la mujer respecto de los del hombre. Esto es, impedir el menoscabo o la desventaja de la mujer respecto del hombre en la consagración y el goce de los derechos;

CENTESIMOQUINTO: Que todo lo señalado respecto de la prohibición de discriminación entre hombres y mujeres no significa que el ordenamiento jurídico no pueda establecer diferencias entre ambos sexos. La igualdad entre hombres y mujeres no puede ser absoluta, incluso para resguardar el propio beneficio de la mujer. Como lo señala un reciente estudio: "el legislador puede establecer diferencias entre los hombres y las mujeres en el contenido de las normas, siempre y cuando existan diferencias reales y efectivas que discriminen a uno de los sexos en ámbitos concretos de la realidad vital; y significa también que el legislador no puede establecer diferencias únicamente en razón del sexo. El ser hombre o el ser mujer, o las diferencias que otrora existieron entre los sexos, no pueden ser susceptibles de diferencias en el contenido de la norma, pues el sexo no constituye per se una diferencia objetiva ni razonable, como tampoco son objetivas y razonables las diferencias pasadas que existieron entre hombres y mujeres 
en ámbitos concretos de la realidad vital." (Cristina Zoco Zabala: Igualdad de género en la nueva normativa nacional y estatutaria a la luz de las innovaciones del ordenamiento comunitario, en: Revista Aragonesa de Administración Pública, 30, junio de 2007, p. 255);

CENTESIMOSEXTO: Que la igualdad jurídica entre hombres y mujeres tiene expresiones muy concretas en el goce de los derechos sociales, del derecho a la salud y, específicamente, en lo relativo a las prestaciones de salud, incluyendo los denominados seguros de salud. En el ámbito comunitario europeo, el Consejo de la Unión Europea adoptó un acuerdo, en diciembre de 2004, sobre la ampliación de la igualdad de trato entre hombres y mujeres al acceso de bienes y servicios y a su suministro, en el que se lee, en relación a la cuestión de autos: "Su ámbito incluye los seguros y actividades financieras conexas siempre que sean privadas, voluntarias y ajenas a la relación laboral. En este contexto, la igualdad de trato es un principio rector, por lo cual la inclusión del sexo como factor actuarial no debería dar pie a diferencias entre las primas y las prestaciones de los seguros", agregándose: "En cualquier caso, los costes relacionados con el embarazo y la maternidad no deberán crear diferencias en las primas y prestaciones". (Consejo de la Unión Europea, Comunicado de prensa c/04/350, Bruselas, 13 de diciembre de 2004);

CENTESIMOSÉPTIMO: Que, igualmente, la doctrina comparada ha puesto hincapié en la disconformidad con el derecho fundamental constitucional de igualdad entre hombres y mujeres, de la diferencia de primas o cotizaciones en los sistemas de seguros de salud. En un artículo se sostiene que "la diferencia de primas entre hombres y mujeres en los seguros privados de salud se relaciona directamente con el sexo de los asegurados, y no con la apreciación de riesgo de hombres y mujeres, pues su monto no se calcula individualmente, sino a partir de su sexo. Así los asegurados son discriminados directamente por su sexo". (Michael Wrasse y Susanne Baer: Unterschiedliche Tarife für Männer und Frauen in den Privaten Krankenversicherung-ein Vortoss gegen den Gleichheitssatz des Grundgesetzes (Diferentes primas para hombres y mujeres en los seguros privados de salud. Una vulneración de la igualdad constitucional de la Ley Fundamental): NJW, 23, 2004, pp. 1623-1627);

CENTESIMOCTAVO: Que el artículo 38 ter de la Ley $\mathrm{N}^{\circ} 18.933$ incluye, en su inciso segundo, tres tipos de diferenciaciones o criterios para que las tablas de factores determinen los tipos de beneficiarios: sexo, condición de cotizante o carga y los rangos de edad;

CENTESIMONOVENO: Que de entre los tres criterios enunciados, debe distinguirse sexo y edad, por una parte, y condición de cotizante o carga, por la otra. Mientras que esta última obedece a una situación en la que cada persona se coloca contractualmente en el marco de la autonomía de la voluntad, el sexo y la edad son factores cuya existencia y evolución transcurren independientemente de la voluntad de las personas. Según el Diccionario de la Lengua Española, sexo significa "condición orgánica que distingue al 
macho de la hembra en los seres bumanos, en los animales y en las plantas", mientras que edad significa "tiempo que ha vivido una persona o ciertos animales y vegetales". Ambas expresiones, por lo tanto, denotan estados naturales, inimputables a las personas que los viven, especialmente para ser afectados negativamente por la ley;

\section{EL DERECHO A LA PROTECCIÓN DE LA SALUD. \\ [...]}

CENTESIMODECIMOCUARTO: Que los principales derechos sociales que la Constitución asegura a todas las personas son configurados a partir de la posibilidad de acceder a una determinada prestación. Así sucede con el derecho a la protección de la salud, en que se debe proteger "el libre e igualitario acceso a las acciones" (artículo $19, \mathrm{~N}^{\circ} 9^{\circ}$ ); lo mismo sucede con el derecho a la seguridad social, en que la acción del Estado debe estar dirigida "a garantizar el acceso de todos los habitantes al goce de prestaciones" (artículo 19, $\mathrm{N}^{\circ} 18^{\circ}$ );

CENTESIMODECIMOQUINTO: Que la Constitución garantiza el acceso a dichas prestaciones asignándole roles al Estado y a los particulares;

CENTESIMODECIMOSEXTO: Que la Ley Fundamental le asigna al Estado un rol de control o supervigilancia. Así, establece que al Estado le corresponde el "control de las acciones relacionadas con la salud" (artículo 19, $\mathrm{N}^{\circ} 9^{\circ}$ ) y, tratándose del derecho a la seguridad social, dispone que "el Estado supervigilará el adecuado ejercicio del derecho a la seguridad social" (artículo 19, $\mathrm{N}^{\circ} 18^{\circ}$ );

CENTESIMODECIMOSÉPTIMO: Que, a su vez, la Constitución le garantiza a los particulares el derecho a elegir que las prestaciones les sean entregadas por entidades estatales o por entidades privadas. Así, en el derecho a la salud esto se expresa en que "cada persona tendrá el derecho a elegir el sistema de salud al que desee acogerse" (artículo 19, $\mathrm{N}^{\circ} 9^{\circ}$ ). Por otra parte, la Carta Fundamental garantiza que no exista monopolio prestacional del Estado. Estas prestaciones, entonces, pueden ser entregadas por "instituciones públicas o privadas" (artículo $19, \mathrm{~N}^{\circ} \mathrm{s} 9^{\circ}$ y $18^{\circ}$ );

CENTESIMODECIMOCTAVO: Que, tratándose del derecho a la salud, la Constitución va más allá, pues establece el "deber preferente del Estado de garantizar la ejecución de las acciones de salud". Esta expresión es utilizada únicamente en este derecho (Silva Bascuñán, Alejandro; Tratado de Derecho Constitucional, Tomo XII, Editorial Jurídica de Chile, Santiago, 2008, p. 140); la Constitución no califica de igual manera otros deberes del Estado (Bulnes Aldunate, Luz; El derecho a la protección de la salud en la Constitución de 1980; en Gaceta Jurídica No 294, diciembre, 2004, p. 18);

CENTESIMODECIMONOVENO: Que este deber del Estado está asociado a que la Constitución no garantiza el derecho a la salud, sino que el derecho "a la protección de la salud"; 
CENTESIMOVIGÉSIMO: Que la fórmula constitucional parte por señalar que es "deber del Estado" dicha protección. Los deberes se ubican entre las situaciones pasivas, junto a la sujeción, a la obligación y a la carga. A diferencia de una obligación, que posee un contenido determinado y concreto, la conducta o actividad en que consiste el deber aparece descrita de modo abstracto y genérico. Además, la obligación tiende a servir el interés de un sujeto concreto, en tanto que el deber público es una conducta impuesta en interés general o de la colectividad. Asimismo, la obligación tiene como correlato un derecho subjetivo, lo que no existe en el deber público (Santamaría Pastor, Juan Alfonso; Principios de Derecho Administrativo General, Editorial Iustel, Madrid, 2006, p. 437);

CENTESIMOVIGESIMOPRIMERO: Que, en seguida, la Constitución utiliza la expresión "preferente", lo que en doctrina ha sido interpretado en el sentido de que el Estado "no es el único obligado a cumplir el deber que se le entrega, sino que él es el principal responsable" (Silva Bascuñán, A; ob. cit., p. 140). Se trata de un deber prioritario y preeminente (Nogueira Alcalá, Humberto; Derechos fundamentales y garantías constitucionales, Tomo III, Editorial Librotecnia; Santiago, 2009, p. 127). El Estado no puede jamás dejar de cumplir esta función (Cea, José Luis; Derecho Constitucional chileno, tomo II; Ediciones Universidad Católica, Santiago, 2004, p. 310). El Estado no puede siquiera poner en riesgo la ejecución de dichas acciones respecto de la población (Nancuante Almonacid, Ulises, y Sotomayor Klapp, Roberto; El derecho de la salud; Editorial Conosur, Santiago, 2001, p. 24). El establecimiento de este deber "significa que el sector privado asume un rol subsidiario en relación con la actividad estatal y no como se entiende en el contexto del orden público económico que ampara la Constitución, en que el Estado se repliega o limita ante el principio de la libre iniciativa o del derecho de toda persona de desarrollar cualquier actividad económica, permitiendo así que los particulares asuman un rol de promotores de esta última" (Vásquez Márquez, José Ignacio; El déficit garantístico del artículo 19 Nº de la Constitución; en XXXIV Jornadas de Derecho Público, Editorial LexisNexis, Santiago, 2004, p. 397);

CENTESIMOVIGESIMOSEGUNDO: Que, a continuación, la Constitución utiliza la expresión "garantizar". Con ello, se apunta a dar seguridad o certeza de que se ejecuten las pertinentes acciones de salud (Silva Bascuñán, A.; ob. cit., p. 140). Para el profesor Cea Egaña, se busca "asegurar o dar certeza, a cualquier titular del derecho, que las acciones de salud le serán proporcionadas, sin discriminación, cuando necesite acceder a una o más de ellas" (ob. cit., p. 310). Se pretende que todo titular del derecho, sin diferencias arbitrarias, podrá efectivamente gozar de las prestaciones de salud (Nogueira Alcalá, H.; ob. cit., p. 127);

CENTESIMOVIGESIMOTERCERO: Que, conforme a la Constitución, dicho deber preferente debe llevarse a cabo, además, "en las formas y condiciones que la ley determine". Ello, sin embargo, no implica una reserva legal absoluta o particularmente intensa, pues la Carta Fundamental establece que sólo "las materias básicas relativas al régimen 
previsional y de seguridad social" son materias de ley (artículo $63, \mathrm{~N}^{\circ} 4^{\circ}$ ) y tal razonamiento debe entenderse extendido a la regulación del derecho a la protección de la salud. En tal contexto, tiene cabida la potestad reglamentaria del Presidente de la República o la que corresponde a los organismos reguladores, de acuerdo a las formas y condiciones determinadas por la ley, no por la autoridad administrativa.

Por otra parte, la Constitución encarga al Estado "el control de las acciones relacionadas con la salud", siendo perfectamente legítimo, en consecuencia, que el legislador otorgue, a los efectos del ejercicio de tal deber-atribución, una potestad normativa a la Superintendencia de Salud, en su condición de organismo de la Administración del Estado (artículo 106, D.F.L. Nº 1, de Salud, 2005);

\section{EL DERECHO A LA SEGURIDAD SOCIAL. \\ [...]}

CENTESIMOVIGESIMONOVENO: Que cabe tener presente en este punto las Actas Oficiales de la Comisión de Estudio del Anteproyecto de la Constitución vigente, de las cuales se extrae el pasaje siguiente, que demuestra cuál fue el propósito que se tuvo para introducir la modificación aludida, especialmente en relación con el principio de solidaridad: "El señor Ortúzar (Presidente) pregunta al señor Camiruaga (Asesor de la Superintendencia de Seguridad Social) si podría definirle los principios de universalidad, uniformidad, integralidad, solidaridad y suficiencia, a que se ha hecho referencia. (...) El señor Camiruaga agrega que la solidaridad se manifiesta en un principio fundamental; es de la esencia de la seguridad social. Donde no existe el principio de solidaridad, acota, no se puede hablar de seguridad social, sino de algún sistema de aborro obligatorio o de otro similar. (...) Es un principio totalmente opuesto al sistema del Derecho Civil que parte de la base de la conmutatividad: en una compraventa los valores deben ser equivalentes. Aquí no." (Acta de la sesión 204ª , pp. 4 y 6);”.”. (Sentencia Rol 1218, C. $30^{\circ}$ ).

Con todo, la Comisión optó por no enumerar los principios de la seguridad social, y así quedó recogido en el texto constitucional;

CENTESIMOTRIGÉSIMO: Que, sin embargo, como lo ha sostenido esta Magistratura: "la supresión, en el texto del artículo $19, \mathrm{~N}^{\circ} 18^{\circ}$, de la Constitución, de los principios rectores de la Seguridad Social, incluidos en el inciso tercero del numeral 21 del artículo 1 del Acta Constitucional $\mathrm{N}^{0} 3$, carece de relevancia, pues tales principios configuran la esencia de aquel derecho, de modo que se entienden siempre absorbidos por él, pues de lo contrario perdería su identidad específica”. (Sentencia Rol 1218, C. 30º);

CENTESIMOTRIGESIMOPRIMERO: Que el contenido esencial de la seguridad social se revela en una interpretación sistemática del texto constitucional en el que se recogieron los principios de solidaridad, universalidad, igualdad y suficiencia y unidad o uniformidad, sobre todo si se ven conjuntamente el derecho a la salud (artículo 19, $\mathrm{N}^{\circ} 9^{\circ}$ ) y el derecho a la seguridad social (artículo 19, $\mathrm{N}^{\circ} 18^{\circ}$ ). Así, en primer lugar, el principio de solidaridad se expresa en el deber que tiene el Estado, es decir, la comunidad políticamente 
organizada, de garantizar económicamente el goce de estos derechos. Las expresiones de la Constitución son claras en este sentido, toda vez que se obliga al Estado a "garantizar" el goce de los derechos; asimismo, se faculta al legislador para establecer cotizaciones obligatorias (numerales $9^{\circ}$ y $18^{\circ}$ del artículo 19 de la Constitución);

CENTESIMOTRIGESIMOSEGUNDO: Que, por otra parte, la Constitución incorpora el principio de universalidad subjetiva, pues estos derechos se encuentran asegurados a todas las personas, sin distinciones de ninguna especie. La expresión "todas las personas" no sólo forma parte del encabezado del artículo 19 constitucional, sino que se refleja en el uso de los términos "cada persona", que utiliza su numeral 90, "prestaciones básicas uniformes" y "todos los habitantes", contenidos en su numeral $18^{\circ}$;

CENTESIMOTRIGESIMOTERCERO: Que también la Constitución acoge el principio de universalidad objetiva, pues asegura estos derechos a las personas frente a un rango amplio de riesgos individuales. En el derecho a la protección de la salud se otorgan "acciones de promoción, protección y recuperación de la salud y de rehabilitación del individuo" y la "ejecución de las acciones de salud" (artículo 19, $\mathrm{N}^{\circ} 9^{\circ}$ ). En el derecho a la seguridad social, se asegura "el acceso de todos los habitantes al goce de prestaciones básicas uniformes" (artículo 19, No $18^{\circ}$ );

CENTESIMOTRIGESIMOCUARTO: Que nuestra Constitución, a su vez, establece claramente que las prestaciones que envuelven los derechos en cuestión han de ser igualitarias y suficientes, sea que se otorguen por el Estado o por agentes privados. Las expresiones que en tal sentido emplea el texto constitucional son: "libre e igualitario acceso" (artículo 19, No $9^{\circ}$ ) y "prestaciones básicas uniformes" (artículo 19, No $18^{\circ}$ ). Por su parte, por mandato de la misma Carta Fundamental, el Estado debe "garantizar" los derechos (artículo 19, $\mathrm{N}^{\circ} 9^{\circ}$, inciso cuarto, y $\mathrm{N}^{\circ} 18^{\circ}$, inciso tercero) y/o "supervigilar" (artículo 19, $\mathrm{N}^{\circ} 18^{\circ}$, inciso cuarto), o incluso "coordinar y controlar" (artículo 19, $\mathrm{N}^{\circ} 9^{\circ}$ );

CENTESIMOTRIGESIMOQUINTO: Que, finalmente, la Constitución ha previsto normativamente la unidad del sistema de protección en materia de seguridad social. Dicha unidad está dada por el deber del Estado de "garantizar" el goce del derecho a la protección de la salud y a la seguridad social, sea que lo preste por sí mismo o por sujetos privados (artículo 19, $\mathrm{N}^{\circ} 9^{\circ}$, inciso cuarto, y $\mathrm{N}^{\circ} 18^{\circ}$, inciso tercero). Su papel es el de dirección y regulación de los privados. En materia de salud, le corresponde "coordinar y controlar" las prestaciones (artículo 19, $\mathrm{N}^{\circ} 9^{\circ}$, inciso tercero) y en seguridad social, específicamente le compete "supervigilar" el sistema (artículo 19, $\mathrm{N}^{\circ} 18^{\circ}$, inciso cuarto);

CENTESIMOTRIGESIMOSEXTO: Que la vinculación estrecha entre el derecho a la protección de la salud y el derecho a la seguridad social se ve manifestada en los propósitos de los sistemas de seguridad social, que son los de asegurar a sus beneficiarios "condiciones de vida ante la ocurrencia de riesgos como la desocupación, la vejez, la incapacidad, entre otros, que les impiden a quienes los experimentan obtener, mediante el trabajo, los medios 
indispensables para su subsistencia o la de su grupo familiar." (Mario Verdugo, Emilio Pfeffer y Humberto Nogueira: Derecho Constitucional, Editorial Jurídica de Chile, 1997, Tomo I, p. 210).

Como se observa, el factor edad es constituyente de ambas garantías, resultando incompatible con sus respectivos propósitos el que la desprotección frente a los estados de necesidad aumente en la misma medida en que aumentan los años de vida;

CENTESIMOTRIGESIMOSÉPTIMO: Que, a mayor abundamiento, la relación entre el derecho a la seguridad social y el derecho a la protección de la salud se ha visto expresada en las consagraciones jurídicas internacionales, como en el Convenio N ${ }^{\circ} 102$ de la Organización Internacional del Trabajo (OIT), que incluye como "riesgos y contingencias sociales en el ámbito de su protección: enfermedad, maternidad, vejez, invalidez, muerte, cesantía o desempleo, cargas familiares y riesgos profesionales" (citado en: Gobierno de Chile, MINSEGPRES: Doctrina Constitucional del Presidente Ricardo Lagos Escobar (20002006), LOM, Santiago de Chile, 2010, Tomo I, p. 223);

CENTESIMOTRIGESIMOCTAVO: Que, como se señaló por este Tribunal en la sentencia Rol 1287, la definición de la seguridad social ha encontrado sustancioso tratamiento en la doctrina chilena, la que ha profundizado en el detalle de sus componentes esenciales y en el alcance de los mismos. Así entendida como "el conjunto de principios que reconocen a todo ser humano el derecho a los bienes indispensables para prevenir sus contingencias sociales y cubrir sus efectos y que regulan las instituciones requeridas para ello" (Alfredo Bowen Herrera: Introducción a la Seguridad Social, Santiago, Ed. Jurídica de Chile, 1992, p. 97), la seguridad social moderna se sustenta en determinados principios que le otorgan su esencia y que la reciente doctrina nacional ha enumerado como los siguientes: i) respecto de los sujetos amparados: universalidad subjetiva, ii) respecto de las contingencias cubiertas: universalidad objetiva, iii) respecto de las prestaciones: igualdad, integridad o suficiencia, iv) respecto del financiamiento: solidaridad, y v) respecto de la administración: unidad o descentralización, subsidiariedad (Héctor Humeres Noguer: Derecho del Trabajo y de la Seguridad Social, Editorial Jurídica de Chile, Santiago de Chile, 2005, Tomo III, pp. 31 y ss.);

CENTESIMOTRIGESIMONOVENO: Que en la misma sentencia citada, esta Magistratura estableció la relación existente entre el derecho a la seguridad social y el derecho a la protección de la salud en el derecho chileno sobre la base de constatar que "en el artículo 92 del DL. $N^{0}$ 3.500, de 1980, contempló la cotización del 7\% de las remuneraciones imponibles de los trabajadores para contribuir al financiamiento de las prestaciones exigidas para la satisfacción de uno y otro de esos atributos esenciales de la persona";

\section{LA DILUCIDACIÓN DEL EXAMEN DE CONSTITUCIONALIDAD.}

CENTESIMOCUADRAGÉSIMO: Que esta Magistratura declaró en el considerando quinto del auto cabeza de este proceso: "Que el artículo 38 ter transcrito 
precedentemente ha sido declarado inaplicable por este Tribunal Constitucional en cuatro sentencias recaídas en las causas de roles $\mathrm{N}^{\circ}$ s 976, 1218, 1287 y 1273. En ellas, esta Magistratura ha declarado que la aplicación del citado precepto, en las respectivas gestiones pendientes, resulta contraria a la Constitución, al vulnerarse los derechos asegurados en los números $2^{\circ}, 9^{\circ}$ y $18^{\circ}$ de su artículo $19^{\prime \prime}$;

CENTESIMOCUADRAGESIMOPRIMERO: Que al dilucidar la cuestión de constitucionalidad que ocupa a esta Magistratura, resulta conveniente reiterar el significado de la declaración de inconstitucionalidad, ya descrito en el apartado I, A de esta sentencia. Los reputados catedráticos Eduardo García de Enterría y Tomás-Ramón Fernández han descrito la institución con una breve formulación: "En general, es evidente, el problema de inconstitucionalidad habrá que resolverlo por un simple contraste lógico de compatibilidad entre dos normas abstractas, la constitucional y la que es objeto de juicio" (Eduardo García de Enterría y Tomás-Ramón Fernández, Curso de Derecho Administrativo I, Thomson Civitas, Navarra, decimocuarta edición, 2008, p. 171);

CENTESIMOCUADRAGESIMOSEGUNDO: Que tratándose de un examen abstracto, esto es, sin referencia a casos concretos, como aquel que se debe desarrollar en el juicio de inaplicabilidad, y que, además, recae sobre derechos fundamentales, como los garantizados en los números $2^{\circ}, 9^{\circ}$ y $18^{\circ}$ del artículo 19 de la Constitución, el que se ha propuesto realizar esta Magistratura respecto del artículo 38 ter de la Ley $\mathrm{N}^{\circ} 18.933$ debe ponderarse según el criterio de razonabilidad, teniendo presente sus dimensiones de adecuación o idoneidad, de necesidad y de proporcionalidad, a las que también aludió el señor Presidente de la República en el informe evacuado en estos autos;

CENTESIMOCUADRAGESIMOTERCERO: Que, consecuentemente con el método descrito, en el caso de autos esta Magistratura ha examinado si el artículo 38 ter de la Ley $\mathrm{N}^{\circ} 18.933$, objeto de este proceso constitucional: a) cumple con ser adecuado a los fines constitucionales de tutelar la igualdad ante la ley, especialmente entre hombres y mujeres, de proteger la salud de las personas incorporadas al sistema privado de salud en el cual actúan las Instituciones de Salud Previsional, especialmente en lo que concierne al rol preferente del Estado en garantizar la ejecución de las acciones de salud y en proteger el libre e igualitario acceso a ellas de todas esas personas, y de asegurar que las personas accedan al goce de las de prestaciones básicas uniformes de seguridad social, garantizadas por la acción del Estado; b) cumple con ser indispensable para alcanzar los fines señalados; y c) si guarda proporcionalidad con tales objetivos;

CENTESIMOCUADRAGESIMOCUARTO: Que, como corolario del análisis efectuado, este Tribunal ha logrado convicción en cuanto a que los números 1, 2, 3 y 4 del inciso tercero del artículo 38 ter de la Ley $\mathrm{N}^{0} 18.933$ no cumplen los supuestos descritos en el considerando precedente y, por consiguiente, son incompatibles con el derecho a la igualdad ante la ley, especialmente entre hombres y mujeres, y lesionan, asimismo, el derecho a la protección de la salud y el derecho a la seguridad social, en 
el sentido que todos ellos se encuentran reconocidos y asegurados en nuestra Carta Fundamental;

CENTESIMOCUADRAGESIMOQUINTO: Que, en primer lugar, es dable observar que si bien las diferencias fundadas en los criterios de la edad y del sexo de las personas, no son, en sí mismas, jurídicamente reprochables, ni tampoco prima facie arbitrarias, siempre que respondan a una fundamentación razonable, sí lo son aquellas que se derivan de dichos preceptos. En efecto, los numerales 1, 2, 3 y 4 del inciso tercero del artículo 38 ter de la Ley $\mathrm{N}^{\circ} 18.933$ son contrarios a la igualdad ante la ley asegurada en el numeral $2^{\circ}$ del artículo 19 de la Carta Fundamental, toda vez que admiten el establecimiento de diferencias arbitrarias al no instituir límites idóneos, necesarios, proporcionados y, por ende, razonables, respecto del ejercicio de la potestad discrecional que el mismo precepto legal le entrega a la Superintendencia del ramo para determinar, a través de "instrucciones de general aplicación", los topes de edad, dentro de la estructura de las tablas de factores que, a su vez, deben utilizar las Isapres al elaborar los planes de salud que ofrezcan a sus afiliados y para determinar la manera cómo influirá en la variación del precio de tales contratos el aumento o la reducción del factor que corresponda a un beneficiario del respectivo plan en razón de su edad;

CENTESIMOCUADRAGESIMOSEXTO: Que, a mayor abundamiento, como ya constató esta Magistratura -considerandos sexagesimoprimero y sexagesimosegundo de esta sentencia- y lo reconoció el señor Presidente de la República en el informe evacuado en este proceso - principalmente en la afirmación que formula la autoridad a fojas 91, con letra destacada, en el punto 3.7. del Capítulo IV del documento-, resulta desproporcionada y carente de razonabilidad la norma contenida en el artículo segundo transitorio de la Ley $\mathrm{N}^{\mathrm{0}} 20.015$, y con apoyo en el mismo juicio jurídico debe estimarse inconstitucional la parte del articulado permanente de la ley en examen que entrega amplia libertad de actuación a un organismo de la Administración en los términos señalados, ya que esa clase de disposiciones se aparta de la razonabilidad que debe presidir la regulación de los derechos fundamentales, como lo recuerdan la doctrina y la jurisprudencia citadas en el cuerpo de este fallo;

CENTESIMOCUADRAGESIMOSÉPTIMO: Que la expresión arbitraria que usa nuestra Constitución en varias de sus disposiciones, entre éstas, en el numeral $2^{\circ}$ de su artículo 19, encontró una precisión jurisprudencial en sentencia de la Corte Suprema de 15 de junio de 1988, que refuerza este parecer al admitir que "la ley pueda hacer diferencias entre grupos siempre y cuando no sea una discriminación arbitraria, esto es, contraria a la ética elemental o que no tenga una justificación racional" (citada en Alejandro Silva Bascuñán, ob. cit., p. 124);

CENTESIMOCUADRAGESIMOCTAVO: Que, además, como lo ha señalado esta Magistratura en sentencias recientes -roles 1273 y 1348-, la diferenciación por sexo y edad que permite el artículo 38 ter de la Ley $\mathrm{N}^{\circ} 18.933$, establece un trato desigual 
para igualdades esenciales, como son: i) la igualdad en dignidad y derechos con que las personas nacen (inciso primero del artículo $1^{\circ}$ de la Constitución), ii) la igualdad de oportunidades como derecho de las personas en la participación en la vida nacional (inciso quinto del artículo $1^{\circ}$ de la Ley Fundamental), iii) la igualdad ante la ley entre el hombre y la mujer (oración final del inciso primero del número $2^{\circ}$ del artículo 19 de la Constitución), y iv) la igualdad de acceso a las acciones de salud (inciso segundo del número $9^{\circ}$ del artículo 19 de la Constitución);

CENTESIMOCUADRAGESIMONOVENO: Que, en segundo lugar, en relación al derecho a la protección de la salud, debe señalarse que una misma materia no puede ser regulada indistintamente por la ley y por normas administrativas y que, en consecuencia, no le corresponde al legislador legalizar o deslegalizar materias, pues eso lo define el constituyente;

CENTESIMOQUINCUAGÉSIMO: Que, como ya se ha señalado, mientras el artículo segundo transitorio de la Ley $\mathrm{N}^{\circ} 20.015$ estableció, por el plazo de 10 años contados desde la entrada en vigencia de dicha legislación, la relación máxima entre el factor de edad más bajo y el más alto de cada tabla, diferenciada por sexo, señalando que ésta iba a ser de hasta 9 veces, en el caso de las mujeres, y de hasta 14 veces, en el caso de los hombres, el número 4 del inciso tercero del artículo 38 ter de la Ley $\mathrm{N}^{\circ} 18.933$ le entregó tal definición a la Superintendencia del ramo;

CENTESIMOQUINCUAGESIMOPRIMERO: Que si el legislador, en el año 2005, fijó en el citado precepto transitorio la determinación de tales márgenes en el entendido de que éstos se hallan dentro de las "materias básicas" de la seguridad social, él mismo vulneró la Constitución al entregarle su definición a un organismo regulador, esto es, a la Superintendencia del ramo, en la determinación permanente -específicamente en el numeral 4 del inciso tercero del artículo 38 ter bajo control-;

CENTESIMOQUINCUAGESIMOSEGUNDO: Que los primeros cuatro numerales del inciso tercero del artículo 38 ter tampoco cumplen lo dispuesto en el inciso cuarto del numeral $9^{\circ}$ del artículo 19 de la Carta Fundamental, en orden a fijar "las condiciones" del deber preferente del Estado de garantizar la ejecución de las acciones de salud y, por consiguiente, son inconstitucionales;

CENTESIMOQUINCUAGESIMOTERCERO: Que, en efecto, dichos preceptos permiten la operación de un complejo mecanismo de reajustabilidad del precio de los planes de salud que se ofrecen en el sector privado. Así, el precio final del plan de salud que debe pagar el afiliado a una Isapre se determina multiplicando el precio base por el respectivo factor de edad que corresponda al afiliado o a alguno de los beneficiarios, de conformidad a la respectiva tabla de factores (artículo 170, letra m), del D.F.L. $\mathrm{N}^{\mathrm{o}} 1$, de Salud, 2005). Por otra parte, el plan de salud es elaborado por la Isapre, la estructura de la tabla de factores es definida por la Superintendencia a través de instrucciones y 
los factores de cada tabla son libremente determinados por la Isapre. A través de este mecanismo, en consecuencia, no se han fijado directamente por la ley "las condiciones" que le manda establecer la Constitución para garantizar el acceso a la prestación, sino que lo que ha hecho el legislador es dispersar la determinación de tales condiciones en distintos actores. Resulta claro, además, que en tal esquema el papel del afiliado, que es el actor principal del derecho a la protección de la salud, prácticamente desaparece, pues queda constreñido a aceptar o a rechazar lo que la entidad previsional le ofrezca;

CENTESIMOQUINCUAGESIMOCUARTO: Que, en este mismo orden de consideraciones, resulta imprescindible indicar que el contrato que celebra un afiliado con una determinada Isapre no equivale a un mero seguro individual de salud, regido por el principio de autonomía de la voluntad, pues opera en relación con un derecho garantizado constitucionalmente a las personas en el marco de la seguridad social y en que la entidad privada que otorga el seguro, tiene asegurada, por ley, una cotización, o sea, un ingreso garantizado. Así, las normas que regulan esta relación jurídica son de orden público;

CENTESIMOQUINCUAGESIMOQUINTO: Que, por otra parte, el mecanismo de reajustabilidad que opera en este ámbito material es exponencial, pues implica que el precio base del plan se multiplica por el factor determinado en su tabla y, de esta forma, se permite un reajuste indefinido. En efecto, de la regulación contenida en las disposiciones bajo examen se puede concluir que la ley no ha establecido condiciones o parámetros razonables, ya que permite que el precio por el seguro de salud contratado con la Isapre aumente en una dimensión que puede ser equivalente a la confiscación de las rentas de un afiliado.

El seguro de salud que opera en este ámbito tiene por objeto garantizar el acceso a las prestaciones de salud. Por lo mismo, precios desproporcionados en relación a las rentas, determinados en base a factores como el sexo y la edad, ambos inherentes a la condición humana, afectan el libre e igualitario acceso a las acciones de salud que el Estado está obligado a garantizar. Para ajustarse a la garantía constitucional reseñada, el legislador debió establecer parámetros prudentes, dentro de latitudes razonables, al determinar las condiciones a las que debe ajustarse la fijación del precio de un seguro de salud que se contrate con una Isapre.

Por otra parte, dicho mecanismo potencia una discriminación en contra de las mujeres, los adultos mayores y los niños menores de dos años, que no tiene justificación racional y, por lo tanto, no se aviene a la Constitución;

CENTESIMOQUINCUAGESIMOSEXTO: Que en el inciso cuarto del numeral $9^{\circ}$ de su artículo 19, la Constitución faculta a la ley para establecer "cotizaciones obligatorias", las que son administradas por "instituciones públicas o privadas". Se les asegura, por tanto, a las Isapres, un ingreso constante. Frente a esa regla el legislador se encuentra obligado a ponderar la obligación del trabajador (afiliado-cotizante) de pagar tales cotizaciones obligatorias y, al mismo tiempo, propender al cumplimiento del deber de asegurar la 
"protección de la salud", previsto en el inciso primero de la misma disposición constitucional. No obstante, el mecanismo de reajustabilidad definido por el legislador en esta materia genera un desequilibrio entre el cobro de las cotizaciones y la protección del derecho a la salud, que deja a este último sin la tutela que la Constitución exige;

CENTESIMOQUINCUAGESIMOSÉPTIMO: Que, en síntesis, los numerales 1, 2, 3 y 4 del inciso tercero del artículo 38 ter de la Ley $\mathrm{N}^{\circ} 18.933$ resultan contrarios a la Constitución, en cuanto impiden garantizar el libre e igualitario acceso de las personas a las acciones de promoción, protección y recuperación de la salud y el derecho que les asiste a elegir el sistema de salud al que deseen acogerse, sea éste estatal o privado, ambas garantías previstas en el número $9^{\circ}$ del artículo 19 de la Constitución Política de la República. Además, el aumento desmedido del precio de los planes de salud al que conduce la amplitud no razonable que la disposición en examen permite, especialmente en los tramos de edad superiores, produce la calidad de "cotizante cautivo" que obliga a emigrar a un sistema al que la persona no desea pertenecer, lo que riñe directamente con el texto constitucional;

CENTESIMOQUINCUAGESIMOCTAVO: Que en relación al número 4 citado, debe agregarse que respecto de la sola formulación del precepto impugnado, sin vinculación con el artículo segundo transitorio de la Ley $\mathrm{N}^{\circ} 20.015$, esto es, en su validez como norma permanente habiendo transcurrido el primer período de diez años que este último contempla, cabe la interrogante sobre su conformidad con la exigencia constitucional dispuesta en el inciso cuarto del numeral $9^{\circ}$ del artículo 19 citado. Esto es, como esta Magistratura ya lo ha expuesto, apreciar si el grado de discrecionalidad que el precepto bajo control le entrega a la Superintendencia de Salud para actuar en la materia referida, corresponde a la envergadura de los bienes jurídicos que su ejercicio abarca o cubre. En efecto, la forma y condiciones como se prestan las acciones de salud, que es la materia cobijada en la reserva legal que nos ocupa, se refiere a un deber del Estado que está destinado a posibilitar que se cumpla la garantía del derecho a la protección de la salud de las personas. Por lo tanto, sin pretender que el legislador determine en este caso un tipo de actividad administrativa plenamente reglada, como se denomina en doctrina, las referidas forma y condiciones de la ejecución de las acciones de salud sí deben determinarse por la ley con un grado de precisión tal que impida que el ejercicio de la discrecionalidad administrativa se extienda a la vulneración de los derechos constitucionales;

CENTESIMOQUINCUAGESIMONOVENO: Que, en tercer lugar, en relación al derecho a la seguridad social garantizado en el artículo $19, \mathrm{~N}^{\circ} 18^{\circ}$, de la Constitución, en íntima relación con el derecho a la protección de la salud ya analizado, es necesario resaltar que también resulta infringido por los preceptos legales en análisis. Lo anterior ocurre, toda vez que, no estableciéndose en la fijación de la relación máxima entre el más alto y el más bajo de los factores de cada tabla que surge del número 4 del inciso tercero del artículo 38 ter, ninguna precisión ni regla especial para las personas que se 
encuentran en situación de obtener las prestaciones de seguridad social, se vulnera su propósito esencial: "La Seguridad Social tiene su razón de ser en que los administrados están sujetos a contingencias sociales. La necesidad de proteger de estas contingencias al ser bumano y a los que de él dependen emana de su derecho a la existencia; de la obligación de conservar su vida y bacerlo en un nivel digno y acorde con su condición de tal" (Héctor Humeres Noguer: Derecho del Trabajo y de la Seguridad Social, Tomo III, ob. cit., p. 23);

CENTESIMOSEXAGÉSIMO: Que, en efecto, el aumento de los precios al amparo de la norma cuestionada puede traer consigo, y así ocurre en la realidad de muchas personas, una completa imposibilidad de costearlos, ocasionando la indignidad que ello significa para el trascurrir de sus vidas y la evidencia de la falta de concreción de la obligación que el texto de la Constitución le asigna al Estado para dirigir su acción a garantizar el acceso de todos los habitantes al goce de prestaciones básicas uniformes;

CENTESIMOSEXAGESIMOPRIMERO: Que de la vigencia de los números 1, 2, 3 y 4 del inciso tercero del artículo 38 ter citado, se desprende una situación contraria a los principios de solidaridad y de equidad que informan no sólo la seguridad social, sino todo el conjunto de derechos fundamentales sociales garantizados en nuestra Constitución, lo que exige de esta Magistratura declararlos como contrarios a ella;

CENTESIMOSEXAGESIMOSEGUNDO: Que, consecuentemente con lo razonado hasta este punto y advirtiéndose el reconocimiento expreso que ha manifestado en autos el Ejecutivo-órgano colegislador, esta Magistratura constata que la formulación de los números 1, 2, 3 y 4 del inciso tercero del artículo 38 ter, cuyo correlato de este último se encuentra en el artículo segundo transitorio de la Ley $\mathrm{N}^{\circ} 20.015$, como podría encontrarse en cualquiera otra norma que se expidiera al amparo del amplio mandato que entrega, es contraria a la Constitución Política de la República, afectando, en lo que a la causa de autos concierne, a los números $2^{\circ}, 9^{\circ}$ y $18^{\circ}$ de su artículo 19 , y así se declarará;

[...]

Y VISTO, además, lo dispuesto en los artículos $1^{\circ}, 6^{\circ}, 19, \mathrm{~N}^{\circ} \mathrm{s} .2^{\circ}, 9^{\circ}$ y $18^{\circ}, 63$ y 93 , inciso primero, $\mathrm{N}^{\circ} 7^{\circ}$, e inciso decimosegundo y 94 , de la Constitución Política de la República, así como en las disposiciones pertinentes de la Ley No 17.997, Orgánica Constitucional de este Tribunal Constitucional,

SE RESUELVE QUE LOS NUMERALES 1, 2, 3 y 4 DEL INCISO TERCERO DEL ARTÍCULO 38 TER DE LA LEY N 18.933, SON INCONSTITUCIONALES. 


\section{COMENTARIO}

El presente comentario pretende abordar el razonamiento sobre derechos fundamentales del fallo del Tribunal Constitucional sobre la Ley de Isapres contenido en la sentencia rol n ${ }^{\circ} 1710-10$ de 6 de agosto de 2010 .

El fallo declaró inconstitucional los numerales 1, 2, 3 y 4 del inciso $3^{\circ}$ del artículo 38 ter de la Ley de Isapres. Lo hizo fundándose en que tales disposiciones afectaban derechos fundamentales consagrados en la Constitución Política. En particular el derecho de igualdad ante la ley, el de protección de la salud y el derecho a la seguridad social.

Dos observaciones preliminares pueden hacerse sobre el razonamiento del tribunal. En primer lugar, su argumentación en términos extensivos fue contundente, en el sentido de que cualquiera de los razonamientos particulares de afectación de derechos fundamentales habría bastado para declarar inconstitucional a la ley. Existían, por tanto, tres razones distintas de la inconstitucionalidad, que individualmente consideradas hubieran servido para concluir la inconstitucionalidad. En segundo lugar, el Tribunal tuvo la opción de construir su razonamiento de inconstitucionalidad sin incorporar la afectación sustantiva de derechos fundamentales. Ello podría haber sido logrado limitándose a la constatación de la infracción de la reserva de ley que también es mencionada. Existen, por tanto, razones basadas en distribución de competencias entre órganos estatales que hubieran bastado, según la concepción del Tribunal, para justificar la inconstitucionalidad. El Tribunal, sin embargo, tomó el más difícil camino de argumentar que ciertos derechos fundamentales estaban siendo afectados por la vigencia de la Ley de Isapres.

La forma en como el Tribunal construyó en cada caso su argumentación requiere un estudio pormenorizado. La afectación de la igualdad ante la ley (art. 19 no 2 CPol) y, en particular, de la igualdad entre hombres y mujeres fue construida sobre el enunciado del inciso $1^{\circ}$ que establece que "los hombres y mujeres son iguales ante la ley" y el enunciado del inciso $2^{\circ}$ que establece la prohibición de diferencias arbitrarias. El Tribunal concluye que de esos enunciados se derivaba un principio general de igualdad de trato y un deber del Estado de eliminar las desventajas de las mujeres respecto de los hombres en la consagración y en el goce de los derechos. Ello acarrea, en opinión del Tribunal que la categoría del sexo no pueda ser utilizada para afectar negativamente a un sujeto. El Tribunal construye sobre esta base un estándar de justificación para las diferencias basadas en el criterio del sexo. Este estándar es designado por el Tribunal como razonabilidad. Luego, puede derivarse de la opinión del Tribunal que cuando una medida no toma en cuenta su efecto sobre el goce de los derechos y afecta negativamente a un sujeto sobre la base de distinguir sobre el criterio de sexo, estaría traspasando el umbral fijado por el estándar de la razonabilidad. Eso es lo que pasa, conforme al considerando 145 en el caso de la Ley de Isapres. Cabe destacar que el Tribunal es cuidadoso en excluir de sus conclusiones la prohibición de la utilización del criterio de sexo. Lo que si hace es fijar un estándar, ambiguo por cierto, para su utilización.

En el caso de la afectación del derecho a protección de la salud (art. 19 n 9 CPol) y del derecho a la seguridad social (art. $19 \mathrm{n}^{\circ} 18 \mathrm{CPol}$ ) son construidos sobre una misma base. En primer lugar, sobre la afectación de la reserva de ley que está establecida en 
ambos numerales (en los incisos $4^{\circ}$ y $2^{\circ}$, respectivamente) y que a juicio del Tribunal no se habría respetado. Sobre esa base construye, en segundo lugar, su razonamiento sobre la afectación de estos derechos, para lo cual recurre a la idea de que estos derechos sociales pueden ser afectados, pues tienen un contenido sustantivo en cuanto derechos fundamentales, lo cual es la idea más revolucionaria del fallo.

En el caso del derecho a la salud, el Tribunal considera que se ven afectados dos aspectos del derecho: el libre e igualitario acceso a las prestaciones de salud y la libertad de elegir entre los sistemas públicos y privados de salud. La afectación en estos casos es sustantiva. Las consecuencias de la norma legal son tales que impiden el goce del derecho consagrado en la Constitución. Las mujeres en edad fértil y las personas de la tercera edad no pueden tener acceso, por causa de tal disposición, a las prestaciones de salud que necesitan y se ven obligadas, contra su voluntad, a dejar el sistema de salud privado por el alto costo que ello significa para las personas en sus condiciones.

El caso del derecho a la seguridad social es en particular interesante. El razonamiento del Tribunal se basa principalmente en que la solidaridad es una característica que está implícita en el concepto de seguridad social. Luego, el derecho a la seguridad social requiere, para asegurar el acceso a las prestaciones mínimas de seguridad social, tanto en un régimen público como en uno privado, tener un contenido solidario. Esa es la única forma en que el sistema no perjudicaría, como actualmente lo hace, a un importante número de sujetos. En la medida que los precios de los planes pueden aumentar de la manera en que lo hacen, y a causa de ello muchos ciudadanos no pueden acceder a sus prestaciones de seguridad social, el derecho fundamental respectivo es afectado. Lo que más llama la atención de este razonamiento es que el Tribunal se aparta de la tradicional e imperante fórmula interpretativa que intenta encontrar en la historia de las disposiciones su contenido y alcance. En este caso, el Tribunal no sólo no recurrió al método histórico sino que abiertamente lo contradijo. Sostuvo que a pesar de que los redactores quisieron excluir el componente solidario de la Constitución él era parte inmanente e inalienable del concepto de seguridad social.

En conclusión, la Ley de Isapres y en particular la autorización a la administración para la fijación de la tabla de factores tal y como estaba establecida era inconstitucional: (1) por discriminar en forma no razonable; (2) por incumplir el mandato de reserva de ley; y (3) por afectar de manera sustantiva el goce de los derechos a las prestaciones de salud y a la seguridad social.

La relevancia del fallo puede analizarse en dos niveles. En un primer nivel, puede sostenerse que el fallo tendrá influencia en la configuración futura del sistema de salud, en particular influenciará la decisión sobre el rol que tendrán en él las isapres y las reglas bajo las cuales la cuota del mercado que se les asigne se desenvolverá. En un segundo nivel, el fallo puede analizarse desde el punto de vista de su comprensión del rol del Estado y de los derechos fundamentales en relación con el disfrute de bienes básicos por parte de los ciudadanos. Desde esta segunda aproximación el fallo viene a expresar un cambio de paradigma constitucional en cuanto a reconocer los derechos sociales como verdaderos derechos fundamentales. Vale la pena una breve reflexión sobre el significado del cambio constitucional que el fallo de las isapres significa y ponerlo en relación con 
el cambio del rol del Estado que mediante la política social de los últimos veinte años se ha llevado a cabo.

El rol del Estado que fue concebido por el gobierno de la dictadura militar y que posteriormente fue heredado al gobierno democrático fue el rol subsidiario y pasivo propio de un Estado neoliberal. Las modificaciones llevadas a cabo por políticas sociales de los gobiernos de la Concertación de Partidos por la Democracia, llevaron a que, mediante la incorporación del Estado como un actor relevante en la satisfacción de las necesidades básicas de la población debamos hablar de un Estado prestacional o Estado social para describir el rol que encarna el Estado de Chile en la actualidad.

Esta evaluación política ha tenido un correlato en el nivel de la comprensión del rol que cabe a la Constitución Política y en particular a los derechos fundamentales. La concepción original de la Constitución Política era que los derechos fundamentales fueran comprendidos como derechos liberales pensados para la defensa del statu quo. La aparición de derechos con carácter social que exigían prestaciones de parte del Estado, como la educación la salud o el trabajo, fueron concebidos como directivas que debían ser ejecutadas por el legislador. Sintomático de esta comprensión de los derechos fundamentales es el hecho de que el recurso de protección sólo protege los derechos liberales y los derechos sociales quedan fuera de su cobertura.

Frente a esta concepción tradicional nos encontramos sorpresivamente con que el fallo acoge una perspectiva diferente, la de que los derechos sociales son derechos exigibles y no sólo directivas y que, por lo tanto, el legislador no es libre de configurarlos a su antojo sino que tiene que respetar ciertas exigencias constitucionales al respecto. Esta es una discusión que lleva bastante tiempo siendo desarrollada en el ámbito académico pero que hoy se inicia, a través de este fallo, en el ámbito institucional.

Respecto a esta comprensión de los derechos sociales que el Tribunal Constitucional recoge, hay dos comentarios que parecen ineludibles de cara a la discusión académica que ha precedido al presente fallo.

El primero es que si bien el fallo reconoce a los derechos fundamentales como derechos exigibles, le concede un importante margen de libertad a las autoridades democráticas para su regulación. El Tribunal se preocupa de no fijar el estándar de lo que es razonable concretamente, por ejemplo, sugiriendo qué regulación habría satisfecho el estándar. Simplemente señala que la actual regulación no satisface el estándar, abriendo la puerta a que el legislador insista en la fijación de una tabla de factores que utilice los criterios de edad y sexo, esta vez de una manera razonable. De esta forma, el Tribunal parece responder a los críticos que adelantaban que mediante la fijación constitucional de la actividad prestacional del Estado se produciría una usurpación de las funciones legislativas.

El segundo comentario se dirige a aclarar, frente a las celebraciones anticipadas, lo limitados que son los alcances del fallo. El fallo sólo beneficia a quienes pertenecen a categorías vulnerables, pero que a su vez se encuentran dentro del selecto grupo del $16 \%$ más rico del país que puede permitirse costear un plan privado de salud. Luego, los miembros de la tercera edad y mujeres beneficiados no serán en realidad los más vulnerables de la sociedad, sino aquellos que teniendo un sistema de salud privilegiado 
no quieren perder ese privilegio producto del encarecimiento de los planes. La solidaridad que el Tribunal busca establecer en el sistema privado, por lo tanto, es una solidaridad entre los miembros del $16 \%$ más rico del país, en que las personas de altos ingresos contribuyan a financiar el aumento del riesgo de las mujeres y adultos mayores de altos ingresos.

Para quienes ven en esta medida un triunfo de la justicia social y diagnostican que las políticas sociales en el ámbito de la salud y la protección social han sido impulsadas y puestas sobre la mesa por el Tribunal Constitucional, anticipándose así a los órganos democráticos, hay que recordar lo irrelevante que significa este fallo para el $70 \%$ más pobre de la población de Chile y lo significativo que para ellos son las medidas impulsadas por las autoridades democráticas como el Plan Auge y la reforma previsional que incorporó las pensiones solidarias, en cuya impulsión, por cierto, el Tribunal no tuvo nada que ver. 